\title{
The X-ray emission of the intermediate polar V 709 Cas
}

\author{
D. de Martino ${ }^{1}$, G. Matt ${ }^{2}$, K. Mukai ${ }^{3,4}$, T. Belloni ${ }^{5}$, J. M. Bonnet-Bidaud ${ }^{6}$, L. Chiappetti $^{7}$, \\ B. T. Gänsicke ${ }^{8}$, F. Haberl ${ }^{9}$, and M. Mouchet ${ }^{10,11}$
}

1 Osservatorio Astronomico di Capodimonte, Via Moiariello 16, 80131 Napoli, Italy

2 Dipartimento di Fisica, Universita' degli Studi Roma Tre, Via della Vasca Navale, Roma, Italy e-mail: matt@fis.uniroma3.it

3 Laboratory for High Energy Physics, NASA/GSFC, Code 662, Greenbelt, MD 20771, USA

4 Universities Space Research Association e-mail: mukai@milkyway.gsfc.nasa.gov

5 Osservatorio Astronomico di Brera, Via E. Bianchi 46, 23807 Merate, Italy e-mail: belloni@merate.mi.astro.it

${ }^{6}$ Service d'Astrophysique Saclay, Gif-Sur-Yvette, France e-mail: bobi@discovery.saclay.cea.fr

7 Istituto di Fisica Cosmica del CNR, Via Bassini 15, Milano, Italy e-mail: lucio@ifctr.mi.cnr.it

8 Universitäts Sternwarte Göttingen, Geismarlandstr. 11, 37083 Göttingen, Germany e-mail: boris@uni-sw.gwdg.de

9 Max Planck Institut für Extraterrestrische Physik, Garching, Germany e-mail: fwh@xray.mpe.mpg.de

10 DAEC et UMR 8631 du CNRS, Observatoire de Paris, Section de Meudon, 92195 Meudon Cedex, France

11 Université Denis Diderot, Place Jussieu, 75005 Paris, France

e-mail: martine.mouchet@obspm.fr

Received 25 April 2001 / Accepted 19 July 2001

\begin{abstract}
We present RXTE and BeppoSAX observations of the Intermediate Polar V709 Cas acquired in 1997 and 1998 respectively. The X-ray emission from 0.1 to $30 \mathrm{keV}$ is dominated by the strong pulsation at the rotational period of the white dwarf $(312.8 \mathrm{~s})$ with no sign of orbital or sideband periodicity, thus confirming previous ROSAT results. However, we detect changes in the power spectra between the two epochs. While the second harmonic of the spin period is present during both observations, the first harmonic is absent in 1997. An increase in the amplitude of the spin pulsation is found between 1997 and 1998 together with a decrease in the X-ray flux. The average X-ray spectrum from 0.1 to $100 \mathrm{keV}$ is well described by an isothermal plasma at $\sim 27 \mathrm{keV}$ plus complex absorption and an iron $\mathrm{K}_{\alpha}$ fluorescent line, due to reflection from the white dwarf surface. The rotational pulsation is compatible with complex absorption dominating the low energy range, while the high energy spin modulation can be attributed to tall shocks above the accreting poles. The RXTE spectrum in 1997 also shows the presence of an absorption edge from ionized iron likely located in the pre-shock accretion flow. The variations along the spin period of the partial covering absorber and of reflection are compatible with the classical accretion curtain scenario. The variations in the spin pulse characteristics and X-ray flux indicate that V 709 Cas experiences changes in the mass accretion rate on timescales from months to years.
\end{abstract}

Key words. accretion - binaries: close - stars, individual: RX J0028+59 = V 709 Cas - X-rays: stars

\section{Introduction}

Intermediate Polars (IPs) are a subclass of magnetic Cataclysmic Variables (mCVs) consisting of a weakly magnetized (up to a few MG) and asynchronously rotating $\left(P_{\text {rot }}<P_{\text {orb }}\right)$ white dwarf (WD) which accretes material from a late type, main sequence, Roche-lobe filling secondary star.

Send offprint requests to: D. de Martino, e-mail: demartino@na.astro.it
The magnetic field influences the details of the flow down to the WD poles. In low field IPs, an accretion disc, truncated at the magnetospheric radius, may form and the material flows from the inner edge of the disc along the field lines in an arc-shaped curtain towards the polar regions of the WD (Rosen et al. 1988). A disc-less (or stream-fed) accretion is expected in systems with higher fields, where material accretes directly from the stream flowing from the secondary star. A mixture of streamfed and disc accretion are also possible, where matter 
from the stream overpasses the disc (disc-overflow, Hellier et al. 1995). Different configurations (disc-fed and discoverflow) can be present at different epochs in the same system, due to long term changes in the mass accretion rate (de Martino et al. 1995; Buckley et al. 1996; de Martino et al. 1999).

The inflow of accreting material onto the polar caps produces a stand-off shock, below which material cools via thermal Bremsstrahlung $(k T \sim 5-30 \mathrm{keV})$. Hard X-rays are highly absorbed by cold matter (column densities up to $10^{23} \mathrm{~cm}^{-2}$ ) within the accretion flow (Ishida et al. 1994) and are expected to be reflected by the WD atmosphere (Beardmore et al. 1995; Done et al. 1995; Matt 1999).

Because of their asynchronous rotation, IPs show a wide range of periodicities at the WD spin $(\omega)$, the orbital $(\Omega)$ and sideband frequencies (Warner 1986; Patterson 1994; Warner 1995), whose amplitudes can be wavelength dependent (de Martino 1993). The X-ray periodicities provide direct information on the actual accretion mode (Wynn \& King 1992; Norton et al. 1996), disc-fed systems being dominated by the WD rotational period. The additional presence of periodicities, at the beat (or synodic) $\omega-\Omega$ and orbital $(\Omega)$ frequencies are an indication of disk-overflow accretion. A pure stream-fed system should show pulses at the synodic frequency only (Hellier 1999). To date only V $2400 \mathrm{Oph}$ is a confirmed stream-fed IP (Buckley et al. 1997). Furthermore disc-fed systems show different rotational pulse characteristics: double-peaked $\mathrm{X}$-ray pulses, generally observed in fast rotators such as YY Dra, can originate from the emission of two accreting poles with tall shocks or large footprints (Norton et al. 1999), whilst single-peaked curves, observed in most slow rotators such as FO Aqr, can instead be produced by small accreting regions. It has been suggested (Norton et al. 1999) that such differences are due to the effect of the magnetic field strength, the rapid rotators containing low field WDs (Norton et al. 1999). Unfortunately, for IPs such a proposal is difficult to confirm observationally, due to the lack of detectable optical-IR polarization in most cases and of spectral features of the WD. An outstanding exception is V 709 Cas (Bonnet-Bidaud et al. 2001), the first IP known to date to display the WD atmospheric features, from which a magnetic field strength lower than $3 \mathrm{MG}$ has been estimated.

V709 Cas (RX J0028.8+5917) was identified as an IP from the ROSAT All Sky Survey (Haberl \& Motch 1995; Motch et al. 1996). The X-ray emission was found to be hard $(H R 2=[1.0-2.4] \mathrm{keV}-[0.4-1.0] \mathrm{keV} /[0.4-$ $2.4] \mathrm{keV})=+0.21$ ) and pulsed at a period of $312.8 \mathrm{~s}$ in pointed follow-up PSPC and HRI ROSAT observations (Haberl \& Motch 1995; Norton et al. 1999) (see Table 1 for the history of pointed X-ray observations). Searches in the HEAO-1, UHURU and Ariel V catalogues lead to the identification of V709 Cas with the previously detected hard X-ray sources $1 \mathrm{H} 0025+588,4 \mathrm{U} 0027+59$ and $3 \mathrm{~A} 0026+593$ (Motch et al. 1996). The X-ray timing analysis shows the presence of the dominant $312.8 \mathrm{~s}$ pulsation and its first and second harmonics, with no sign of orbital and sideband variabilities (Norton et al. 1999). Two possible orbital periods at $P_{\Omega}=5.4 \mathrm{~h}$ and $P_{\Omega}=4.5 \mathrm{~h}$ have been inferred from low temporal resolution optical spectroscopy (Motch et al. 1996), each being the one day alias of the other. The former has been recently confirmed spectroscopically (Bonnet-Bidaud et al. 2001) and photometrically (Kozhevnikov 2001). This suggests that V709 Cas is a classical IP: a hard X-ray emitting and disc-accreting $\mathrm{mCV}$.

In this paper we present X-ray observations of V 709 Cas acquired with the RossiXTE (RXTE) and the BeppoSAX satellites. The latter also provides the first simultaneous soft and hard X-ray data which allow a final characterization of its X-ray spectrum and its variability.

\section{Observations and data reduction}

\subsection{The RXTE data}

V709 Cas was observed with RXTE (Bradt et al. 1993) between March 26-28, 1997, over a 2.3 day interval. As the source was not significantly detected with the HEXTE instrument, operating in the energy range $20-200 \mathrm{keV}$, we limited the analysis to the PCA instrument, consisting of an array of 5 proportional counter units (PCU) sensitive in the $2.5-60.0 \mathrm{keV}$ band. The effective on-source exposure time, after standard screening, was $40 \mathrm{ks}$. V 709 Cas was detected at a net count rate of $7.04 \pm 0.01 \mathrm{cts} \mathrm{s}^{-1}$ per PCU in the $2.5-25 \mathrm{keV}$ band. We have estimated the PCA background using a faint source model appropriate for the epoch of the observation. The RXTE data are telemetred in two standard, and up to four additional, data modes. Two separate observation modes were used for spectral and timing analysis. For the production of spectra, we used Standard 2 mode data, which have a time bin size of $16 \mathrm{~s}$. In order to improve the signal to noise, we used only data from the top layers of the PCUs. This reduces the net signal by $20 \%$ and the estimated background by roughly $50 \%$. Since spectral uncertainties, particularly above $10 \mathrm{keV}$, are dominated by systematic uncertainties in the background model, this improves the quality of the net spectrum. For timing analysis, we have used the Event mode data, with a $8 \mathrm{~ms}$ time resolution and 32 PHA channels. As no selection by PCU or PCU layer is possible with this data mode, all layers from all PCUs were used. All analyzed data refer to the effective 2.9-24.2 keV band. The average background level in the five PCU units is $77.1 \mathrm{cts} \mathrm{s}^{-1}$ from all layers, while it is $40.3 \mathrm{cts} \mathrm{s}^{-1}$ from the top layer.

\subsection{The BeppoSAX data}

A later observation with the BeppoSAX satellite (Boella et al. 1997) was carried out between 1998 July 5-7 with the set of the co-aligned Narrow Fields Instruments (NFI), used as prime pointing instruments, covering the wide energy range $0.1-300 \mathrm{keV}$. The source was detected with the LECS $(0.1-10 \mathrm{keV})$, the MECS $(1.3-10 \mathrm{keV})$ and PDS (15-300 keV) detectors at net count rates of 
Table 1. History of pointed X-ray observations of V 709 Cas.

\begin{tabular}{lccccc}
\hline \hline Date & Instrument & $\begin{array}{c}\text { Energy Band } \\
(\mathrm{keV})\end{array}$ & $\begin{array}{c}\text { Count Rate } \\
\mathrm{cts} \mathrm{s}^{-1}\end{array}$ & $\begin{array}{c}\text { Flux } \\
\mathrm{erg} \mathrm{cm}^{-2} \mathrm{~s}^{-1}\end{array}$ & Source \\
\hline 1992, Jul. & ROSAT PSPC & $0.1-2.4$ & 0.60 & $8.8 \times 10^{-12}$ & $a$ \\
1997, Mar. & RXTE PCA & $2-10$ & 5.20 & $6.6 \times 10^{-11}$ & $b$ \\
1998, Feb. & ROSAT HRI & $0.1-2.4$ & 0.26 & $\sim 1 \times 10^{-11}$ & $c$ \\
1998, Jul. & BeppoSAX MECS & $2-10$ & 0.50 & $4.5 \times 10^{-11}$ & $b$ \\
\hline \hline
\end{tabular}

$a$ : Haberl \& Motch (1995).

$b$ : This work.

$c$ : Norton et al. (1999).

Note: The X-ray flux is estimated using the count rate conversion facility PIMMS-HEASARC at GSFC-NASA, adopting $k T=10 \mathrm{keV}$ and $N_{\mathrm{H}}=9.8 \times 10^{20} \mathrm{~cm}^{-2}$.

$0.243 \pm 0.003 \mathrm{cts} \mathrm{s}^{-1}, 0.497 \pm 0.002 \mathrm{cts} \mathrm{s}^{-1}$ and $0.346 \pm$ $0.028 \mathrm{cts} \mathrm{s}^{-1}$ respectively. Effective on-source exposure times have been $35.3 \mathrm{ks}$ for the LECS, $86.9 \mathrm{ks}$ for the MECS and $79.5 \mathrm{ks}$ for the PDS detector (two units, see below).

The LECS light curves and spectra have been extracted using a circular region with a radius of 8 arcmin centred on the source, whilst MECS light curves and spectra have been extracted with a smaller 4 arcmin radius. While the whole LECS band will be used for timing analysis, the spectra from this instrument have been analyzed only below $4 \mathrm{keV}$ due to calibration problems at higher energies. For both intruments, the background has been measured and subtracted using the same detector regions during blank field pointings. The measured background in the LECS $\left(2.74 \times 10^{-2} \mathrm{cts} \mathrm{s}^{-1}\right)$ and MECS $\left(6.95 \times 10^{-3} \mathrm{cts} \mathrm{s}^{-1}\right)$ is much lower than the source count rate.

The PDS is a collimated instrument which monitors the background continuously switching two (of the four) detectors with a dwell time of $96 \mathrm{~s}$. The PDS light curves and spectra have been extracted using a standard routine provided by the BeppoSAX Data Center (SDC). We have conservatively extracted the light curves in the energy range of $15-70 \mathrm{keV}$, above which the source is hardly detected. The average net count rate in this range is $0.270 \pm 0.020 \mathrm{cts} \mathrm{s}^{-1}$. The procedure allows rejection of particle background events, as well as spikes caused by single particle hits, which produce fluorescent cascades which are mainly recorded below $30 \mathrm{keV}$. The background spectrum is evaluated for each of the two half arrays accumulating the off-source spectra. The background light curves are constructed by linear interpolation between two off-source pointings in each array. Then the spectra and light curves from the two arrays are merged together to construct the background spectrum and light curve for final subtraction. The average background count rate is $10.31 \mathrm{cts} \mathrm{s}^{-1}$. We also performed alternative ways to subtract background, accounting for collimator dwell time effects in order to construct the net PDS light curve for periodicity search purposes. We detected no significant modulation in the $15-70 \mathrm{keV}$ energy range (see Sect. 3).
The history of the pointed X-ray observations of V 709 Cas is reported in Table 1.

\section{Timing analysis}

A search for periodicities has been performed on light curves extracted from both data sets in similar energy bands. The range $2.9-9.8 \mathrm{keV}$ has been used for the RXTE PCA data, and the 1.3-10 keV band for the BeppoSAX MECS data. In Fig. 1 the RXTE and BeppoSAX light curves with a temporal resolution of $32 \mathrm{~s}$ are shown. A short term periodicity is clearly apparent, with no indication of variability (periodic or not) on a longer (hours) timescale.

Fourier analysis has been performed on PCA $4 \mathrm{~s}$ and MECS $5 \mathrm{~s}$ binned light curves using the DFT algorithm (Deeming 1975). The corresponding power spectra, shown in Fig. 2 (lower panels), reveal a strong peak at the known $312.8 \mathrm{~s}$ period at both epochs. While the first and second harmonics are observed in the MECS spectrum, the PCA spectrum lacks power at the first harmonic. In order to remove the windowing effects of unevenly sampled data (see spectral windows inserted in the lower panels of Fig. 2), the CLEAN algorithm (Roberts et al. 1987) has been used, adopting a gain of 0.1 and 500 iterations. From the CLEANed power spectra, shown in Fig. 2 (upper panels), neither low frequency (orbital) periodicity nor the beat or other orbital sidebands can be detected.

From the MECS and PCA CLEANed spectra the amplitude of the main signal is $A_{\omega}=0.108 \pm 0.001 \mathrm{cts} \mathrm{s}^{-1}$ and $A_{\omega}=0.82 \pm 0.01 \mathrm{cts} \mathrm{s}^{-1}$ respectively, corresponding to a fractional amplitude of $23 \%$ and $18 \%$ at the two epochs. A larger amplitude in the July 1998 BeppoSAX data is also found for the second harmonic, $A_{3 \omega}=0.034 \pm$ $0.002 \mathrm{cts} \mathrm{s}^{-1}$ (fractional amplitude $7.5 \%$ ), with respect to the March 1997 data, $A_{3 \omega}=0.33 \pm 0.01 \mathrm{cts} \mathrm{s}^{-1}$ (fractional amplitude of $6.5 \%$ ). This also translates in the lack of detection of the first harmonic in the RXTE data while in the BeppoSAX observations the first harmonic is definitively detected, with an amplitude of $A_{2 \omega}=0.019 \pm$ $0.002 \mathrm{cts} \mathrm{s}^{-1}$ (fractional amplitude $3.8 \%$ ). This fullfils the $4 \sigma$ criterium, with $\sigma=4.3 \times 10^{-3} \mathrm{cts} \mathrm{s}^{-1}$ being the average 
RXTE (March 1997)

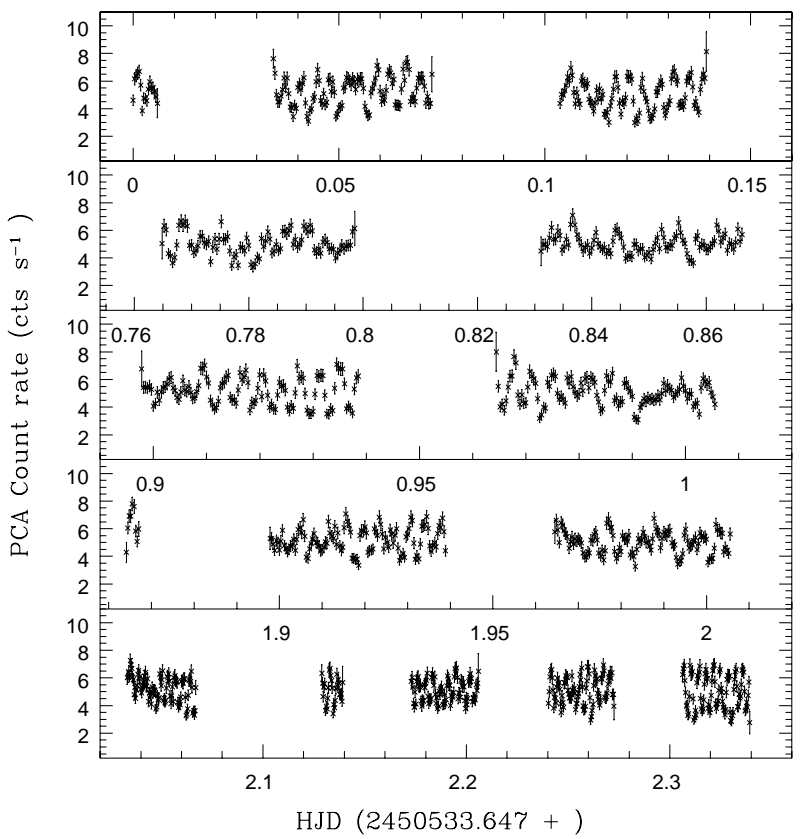

BeppoSAX (July 1998)

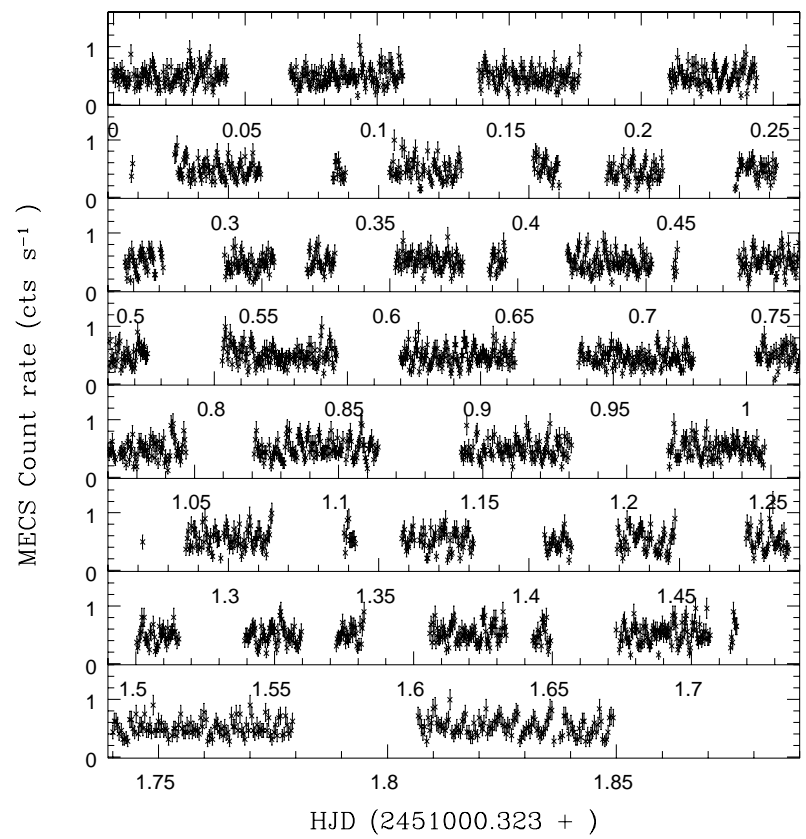

Fig. 1. The net RXTE PCA (2.9-9.8 keV) (left panel) and BeppoSAX MECS (1.3-10 keV) (right panel) $32 \mathrm{~s}$ binned light curves of V 709 Cas in March 1997 and July 1998 respectively.
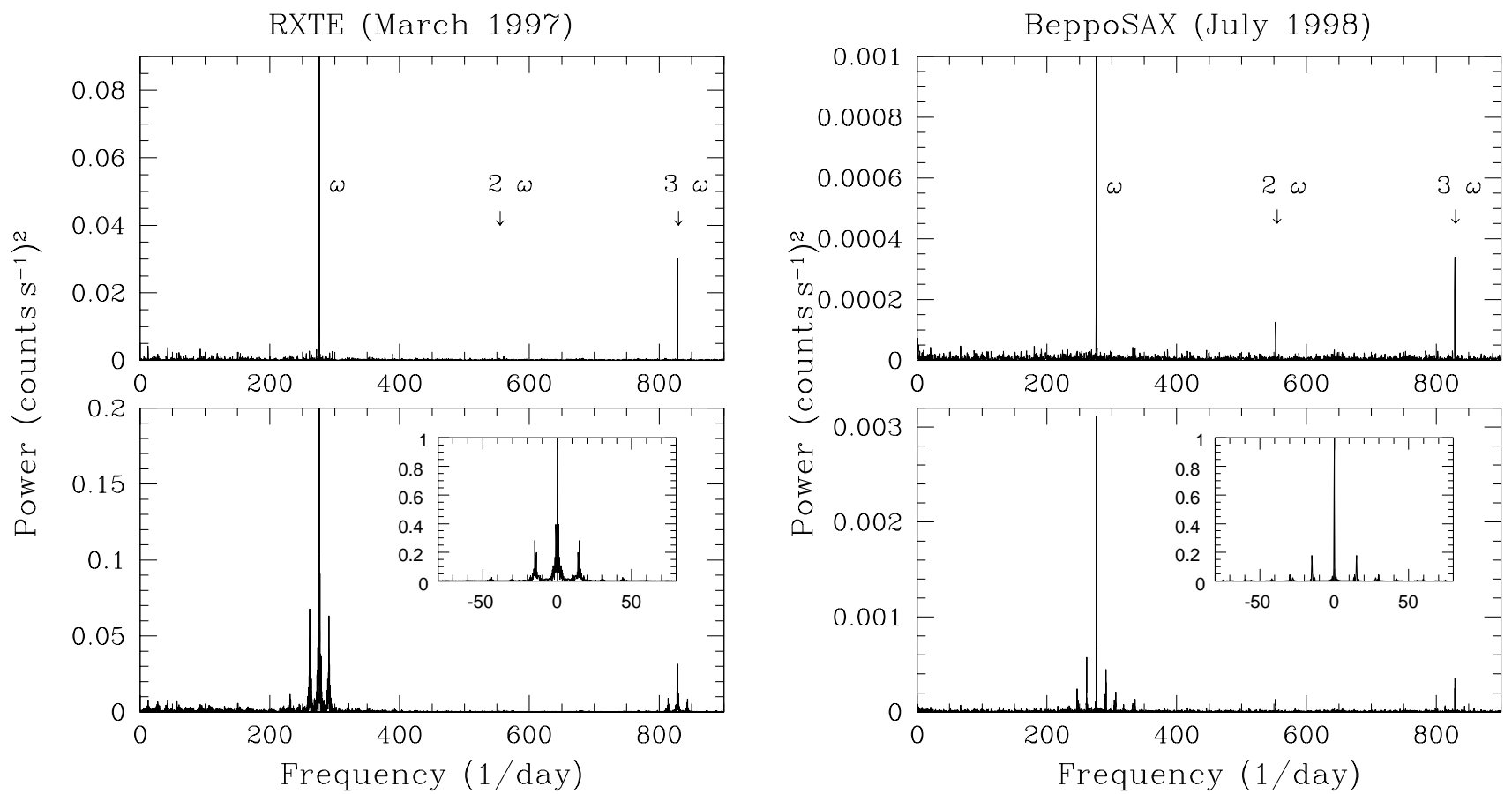

Fig. 2. The PCA (left panel) and the MECS (right panel) power spectra of V 709 Cas. Lower panels: the DFTs show a strong signal at $276.26 \mathrm{~d}^{-1}$. In July 1998 both first and second harmonics are present, while in March 1997 the first harmonic is not detected. The spectral windows are shown in the inserted panels. Upper panels: the removal of windowing effects is shown in the CLEANed spectra.

amplitude from the residual CLEANed spectrum. Here we note that the ROSAT HRI observation carried out in February 1998 (Norton et al. 1999), and hence about four months earlier than the BeppoSAX pointing, also shows the presence of the first and second harmonics. However, the ratios of the power at the fundamental frequency to the first and to the second harmonics in the BeppoSAX LECS $(0.1-2.4 \mathrm{keV})$ range are about 90 and 10 respectively, a factor of three and two larger than those derived from the ROSAT HRI observations.

A sinusoidal fit to the MECS light curve using three frequencies corresponding to the fundamental, first and 

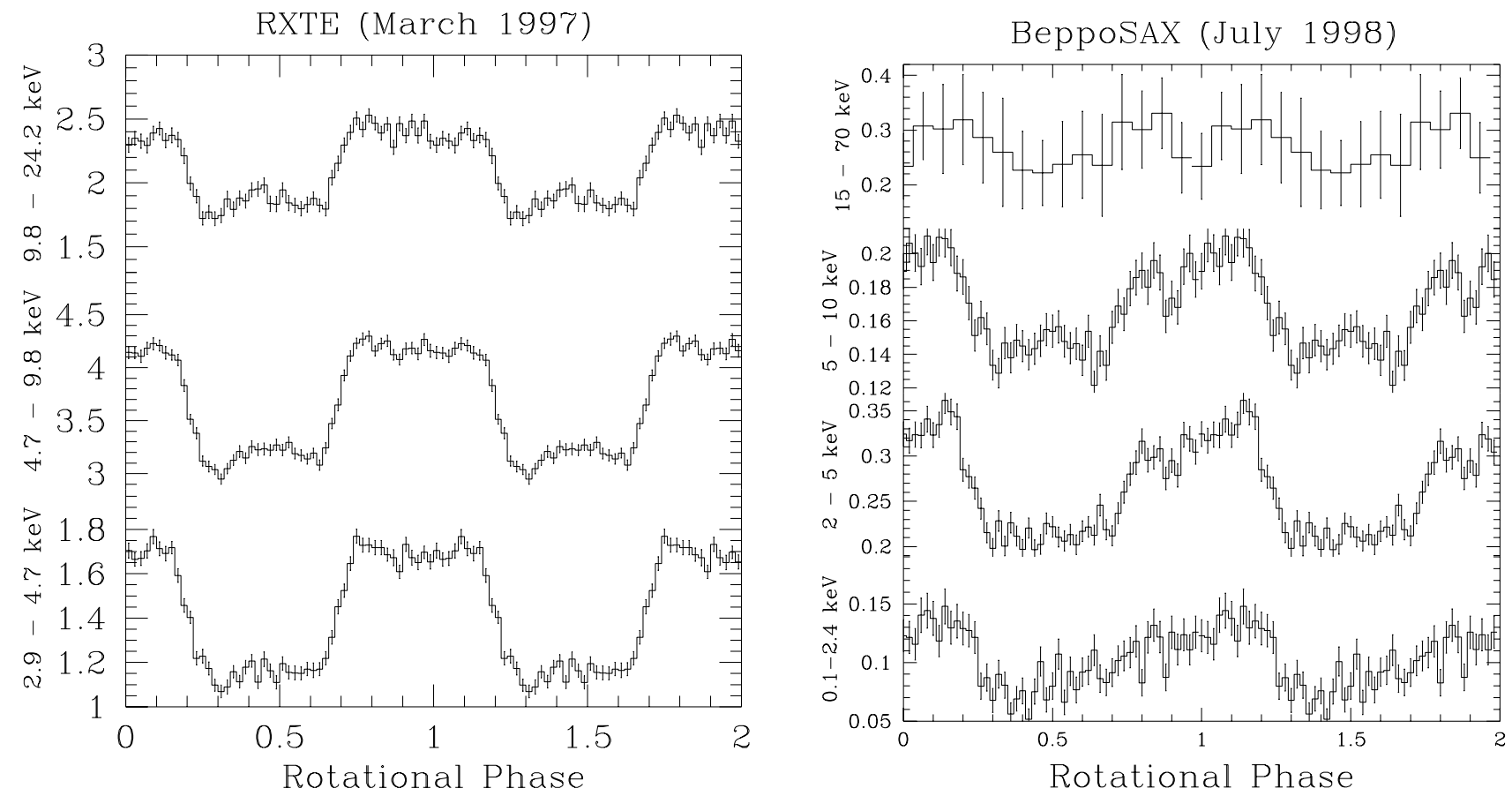

Fig. 3. Left panel: the RXTE light curves in the $2.9-4.7 \mathrm{keV}, 4.7-9.8 \mathrm{keV}$ and $9.8-24.2 \mathrm{keV}$ ranges (from bottom to top) folded along the rotational period using the ephemeris reported in the text. Right panel: the BeppoSAX net spin light curves in the energy ranges (from bottom to top): $0.1-2.4 \mathrm{keV}, 2-5 \mathrm{keV}, 5-10 \mathrm{keV}$ and 15-70 keV. A $6.26 \mathrm{~s}$ bin size has been used except for the PDS (15-70 keV) light curve for which a $21 \mathrm{~s}$ bin has been adopted.

second harmonics yields to a period of $312.77 \pm 0.01 \mathrm{~s}$. Instead, for the PCA light curve two sinusoids, corresponding to the fundamental and second harmonic, have been used, which give $P_{\omega}=312.746 \pm 0.003 \mathrm{~s}$. The present determinations are compatible within errors with the previously determined $312.78 \pm 0.03 \mathrm{~s}$ period from the ROSAT data (Haberl \& Motch 1995; Norton et al. 1999). Times of maxima for each data set are derived: $\mathrm{HJD}_{\max }=$ 2450533.647454(3) and $\mathrm{HJD}_{\max }=2451000.32346(2)$. The accuracy of the derived ephemeris, however, does not allow to bridge the gap between the RXTE and BeppoSAX data sets.

The shape of rotational pulse has been inspected in different energy bands (Fig. 3). The RXTE data in the 2.9-4.7 keV, 4.7-9.8 keV and 9.8-24.2 keV bands (Fig. 3, left panel) have been folded in 50 bins along the rotational period using the corresponding time of maximum. The BeppoSAX data instead have been extracted in the 0.1-2.4 keV LECS range (for a direct comparison with the ROSAT light curve see Fig. 4), in the $2-5 \mathrm{keV}$ and 5-10 keV MECS ranges as well as in the 15-70 keV PDS band. The spin light curves, also folded in 50 bins (except for the PDS where a larger bin size (21 s) has been used), are shown in Fig. 3 (right panel). At both epochs, the rotational pulse is not sinusoidal and shows a broad structured maximum (extending 0.46 in phase). The spin pulse as observed by RXTE has relatively symmetrical rise and decay. On the other hand, the BeppoSAX light curve is highly asymmetrical with a slow rise and a steeper decay. The maximum is not flat with count rates increasing

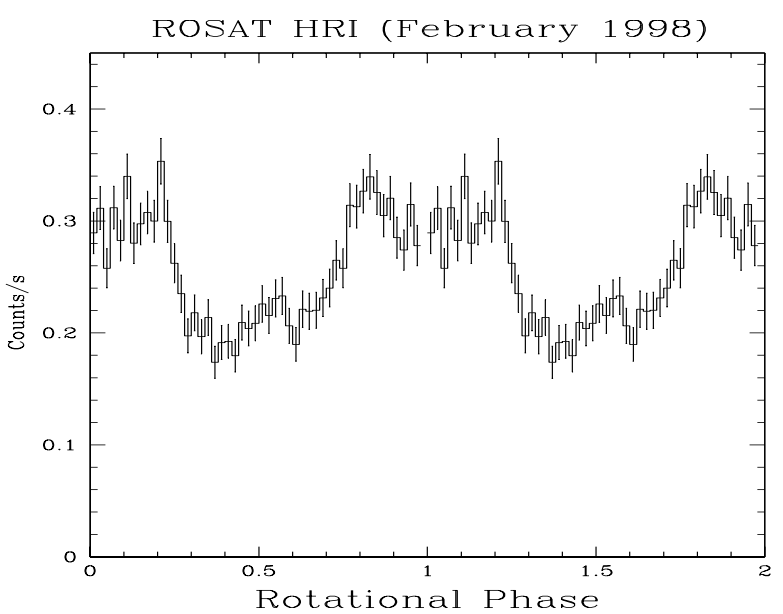

Fig. 4. The ROSAT HRI rotational pulsation in the 0.1$2.4 \mathrm{keV}$ band, observed by Norton et al. (1999) has been folded at the period of $312.77 \mathrm{~s}$ adopting a bin size of $6.26 \mathrm{~s}$. Phase zero is arbitrary (see text).

until $\phi=0.12$. A dip feature with fractional depth $\sim 8 \%$ can be recognized in the hard MECS bands at $\phi=0.9$. This feature does not occur at mid-maximum and it is less pronounced in the soft band. This feature could also be present in the RXTE light curve, but its depth is only marginally significant. The changes in the shape of the spin modulation between the two epochs then explain the presence of the first harmonic in the BeppoSAX data and the lack of such component in the RXTE data. 

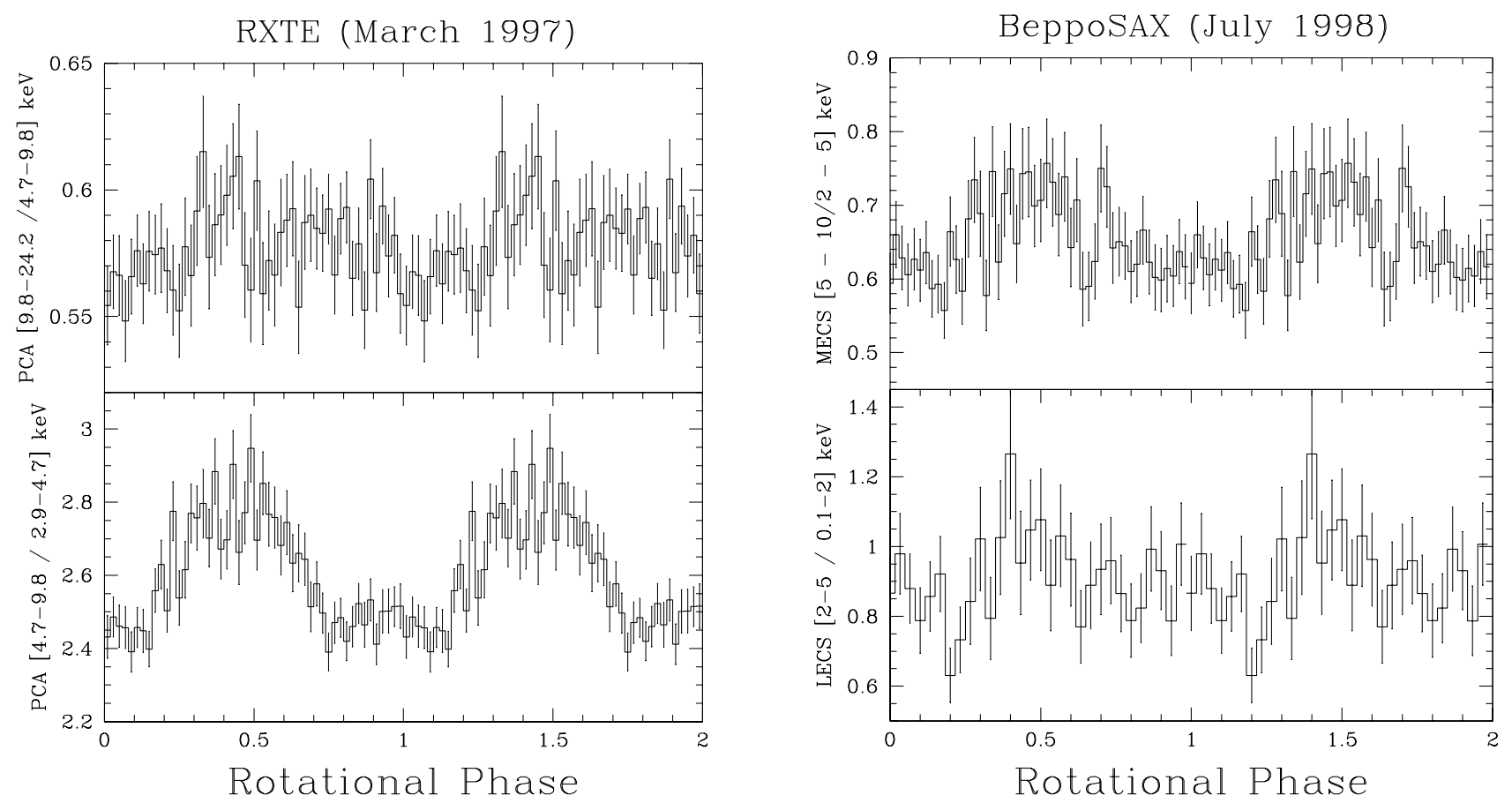

Fig. 5. The behaviour of hardness ratios in the soft and hard X-ray bands along the rotational period. Left panel: the RXTE PCA 5-10 keV and 3-5 keV ratios (lower panel) and the $10-24 \mathrm{keV}$ and 5-10 keV ratios (upper panel). Right panel: the BeppoSAX MECS 5-10 keV and 2-5 keV ratios (upper panel) and the LECS $2-5 \mathrm{keV}$ and $0.1-2 \mathrm{keV}$ flux ratios (lower panel). Both show a spectral hardening at rotational minimum. The effect decreases at increasing energies.

The spin pulsation decreases in amplitude from low to high energies: the pulsed fraction in the RXTE data changes from $46 \%$ in the $2.9-4.7 \mathrm{keV}$ range to $32 \%$ in the $9.8-24.2 \mathrm{keV}$ band, and in the BeppoSAX data it decreases from $86 \%$ in the $0.1-2.4 \mathrm{keV}$, to $56 \%$ in the $2-5 \mathrm{keV}$ and to $52 \%$ in the $5-10 \mathrm{keV}$ band. In the PDS range, the variability is not statistically significant. The light curves reveal an energy dependence of the pulse shape changing from a strongly asymmetrical (at low energies) to a broad (at high energies) structured shape. A comparison of the low energy pulsation in the $0.1-2.4 \mathrm{keV}$ (ROSATlike) band observed in July 1998 with the February 1998 ROSAT HRI rotational curve (Norton et al. 1999) shows a different morphology. The ROSAT spin pulsations is reported in Fig. 4 with an arbitrary phasing since the BeppoSAX ephemeris is not accurate enough. The fractional amplitude of the ROSAT spin pulse is about half that observed by BeppoSAX. Also, the broad maximum in the BeppoSAX data still covers the same phase range but the "double-peaked" shape seen in the ROSAT data, with a secondary minimum centred on the broad maximum, has essentially disappeared. The dip in the high energy light curve does not seem to have a counterpart in the soft X-ray pulse. This feature is not centered on the maximum as observed in the ROSAT light curve. Although a direct comparison of the RXTE and ROSAT light curves should not be performed due to the different energy coverages, the flat-topped RXTE light curve resembles more closely the ROSAT spin pulsation with two almost identical maxima. Again, the detection of an increasing power of the first harmonic in the RXTE, ROSAT and BeppoSAX observations accounts for such differences.

The energy dependence of the rotational pulse manifests itself in the hardness ratios in both soft and hard X-ray bands. In Fig. 5 (right panel), the LECS (2$5 \mathrm{keV} / 0.1-2 \mathrm{keV})$ and MECS $(5-10 \mathrm{keV} / 2-5 \mathrm{keV})$ ratios show a hardening at rotational minimum and a softening at rotational maximum. This behaviour is also observed in the RXTE PCA data (Fig. 5, left panel), which also shows a negligible energy dependence of spin pulses above $10 \mathrm{keV}$. A hardening at pulsation minimum is a typical behaviour in IPs, which is produced by the larger photoelectric absorption when viewing along the accretion curtain. We note that the BeppoSAX hardness ratios do not reveal changes during the maximum phases, which would be expected if the double-humped maximum is due to enhanced absorption effects within one accretion column. As it will be discussed later, the presence of two accreting poles can account for this behaviour. At higher energies a different mechanism responsible for the spin pulsation could be at work as it will be discussed in Sects. 4 and 5 .

\section{Spectral analysis}

An analysis of the time-averaged X-ray spectra of both RXTE and BeppoSAX observations has been performed in order to estimate the basic parameters of the emitting region, in particular the temperature of the optically thin plasma, interstellar and possible circumstellar absorptions and to obtain information on the reprocessing of 
the hard X-rays from the WD surface. The spectral analysis has been performed with the XSPEC fitting package. Henceforth, all quoted errors on spectral parameters refer to $90 \%$ confidence level for the parameter of interest. The BeppoSAX spectra have been fitted between $0.3-70 \mathrm{keV}$, while the RXTE ones between 3.6-20 keV.

\subsection{The BeppoSAX spectra}

A spectral fit consisting of an optically thin plasma (MEKAL model. Mewe et al. 1995 in XSPEC) with a single component absorber does not produce a satisfactory fit $\left(\chi^{2} /\right.$ d.o.f. $\left.=243 / 114\right)$, the temperature being as high as $60 \mathrm{keV}$. The hydrogen column density is $2.6 \times 10^{21} \mathrm{~cm}^{-2}$ which is lower than the total galactic hydrogen column density $\left(4.5 \times 10^{21} \mathrm{~cm}^{-2}\right)$ in the direction of the source. The residuals show an excess of counts in the low energy portion of the spectrum, likely due to a more complex absorption, as well as around $\sim 6 \mathrm{keV}$. Hence we have included a Gaussian $\mathrm{K}_{\alpha}$ irone line centred at $6.4 \mathrm{keV}$ and an additional (partial) absorber. The presence of this absorber is also suggested by the energy dependence of the spin pulse and the strong (see below) iron line. We then obtain a much better fit $\left(\chi_{\nu}^{2}=1.17\right)$ (see Table 2 , model 1$)$. A lower temperature is derived, namely $k T=42 \mathrm{keV}$ which reflects the effects of a complex model fitting. Here we also note that a multi-temperature plasma model (CEMEKL in XSPEC, which is built from the MEKAL code, adopting a power-law temperature profile, Singh et al. 1996) gives a worse fit $\left(\chi_{\nu}^{2}=1.5\right)$, especially for the ionized iron lines and the low energy portion of the spectrum. The hydrogen column density, $N_{\mathrm{H}}=8.7 \times 10^{20} \mathrm{~cm}^{-2}$, is close to that derived from ROSAT data $\left(N_{\mathrm{H}}=9.8 \times 10^{20} \mathrm{~cm}^{-2}\right.$ for a fixed temperature of $k T=10 \mathrm{keV}$, Haberl \& Motch 1995). This can be regarded as an upper limit to the interstellar column density. For the partial absorber we find $N_{\mathrm{H}}=2.92 \times 10^{22} \mathrm{~cm}^{-2}$ and a covering fraction $C_{\mathrm{F}}=0.29$. The equivalent width of the $6.4 \mathrm{keV}$ fluorescent line is $E W=218 \mathrm{eV}$. The metal abundance, left free to vary, is just consistent with the solar value. Given the high temperature of the single temperature plasma model, the metal abundance is only sensititive to the iron abundance. We have adopted a relative iron abundance by number of $4.7 \times 10^{-5}$ (Anders \& Grevesse 1989). The observed flux in the $2-10 \mathrm{keV}$ range is $4.5 \times 10^{-11} \mathrm{erg} \mathrm{cm}^{-2} \mathrm{~s}^{-1}$, a factor 2.5 higher than that extrapolated from the ROSAT 1992 PSPC data, adopting $k T=10 \mathrm{keV}$, and it is similar to other measurements by previous satellites (Motch et al. 1996).

A strong $\mathrm{K}_{\alpha}$ iron line would originate by fluorescence either from the surface of the WD or from the pre-shock accretion column, or both. To account for the large equivalent width, the column density of cold matter should be as high as $2 \times 10^{23} \mathrm{~cm}^{-2}$ (Inoue 1985), while a factor of $\sim 10$ lower is found for the partial absorber. Hence it seems likely that the bulk of the fluorescent line comes from the WD surface. This suggests the presence of the contribution of a Compton reflected continuum from the white dwarf which is expected to go along with the fluorescent iron line (Matt et al. 1991; Done et al. 1992). Therefore, the introduction of a Compton reflection continuum is physically plausible, and turns out to be significant at the $99.7 \%$ confidence level, according to the $F$-test (see Table 2, model 2). Both temperature and fluorescent line parameters change when adding such component: a lower temperature $(27 \mathrm{keV})$ and $E W=179 \mathrm{eV}$ are derived from this fit. Hence, neglecting the reflected contribution the temperature and the strength of the fluorescent line are overestimated. The grand average combined LECS, MECS and PDS spectra fitted with such a model are shown in Fig. 6. The relative normalization of the reflection component, represents the solid angle subtended by the cold matter in units of $2 \pi$ steradians for an average value of $\cos \theta=0.5$ where $\theta$ is the angle between the line of sight and the normal to the reflecting surface. The value 0.92 is compatible with reflection of photons from the WD surface which subtends an angle of about $2 \pi$. The iron and metal abundances (linked together in this fit) are within errors consistent with solar values. Furthermore the partial absorber covering fraction and column density are, as expected, the same as in the previous fit without reflection.

The variability in the hardness ratios is a strong evidence that spectral changes occur at the rotational period. A spin resolved spectral analysis has been performed using only the LECS and MECS data, due to the lack of information on variability from PDS data. Spectra have been extracted in 10 rotational phase bins. In order to estimate changes in the spectral parameters, a fit with a composite model consisting of a MEKAL emission plus the interstellar column density, a partial absorber and Gaussian line has been performed. With the exclusion of the PDS data the temperature of the optically thin gas has been fixed at $30 \mathrm{keV}$, which is the value obtained from fitting the average spectrum without the PDS data to model 1 . We note that the fit quality does not substantially change if $k T=40 \mathrm{keV}$ is assumed. As a first attempt both covering fraction $C_{\mathrm{F}}$ and $N_{\mathrm{H}}$ of the partial absorber and the Gaussian line normalization have been left free to vary, with the remaining parameters fixed at the values found for the phase-averaged spectrum (model 1). In these fits, the partial covering parameters are less well defined, especially $N_{\mathrm{H}}$, which is essentially constant within errors. Hence fits have been performed again varying only $C_{\mathrm{F}}$ and the Gaussian normalization, with $N_{\mathrm{H}}$ of the partial absorber fixed at the value found for model 1 . The results are shown in Fig. 7, where the changes in the covering fraction (a factor $\sim 1.6$ ) indicate an increase in absorption at rotational minimum. The $E W$ of the fluorescent line also increases at rotational minimum by a factor $\sim 2.3$.

An attempt to search for variations in the reflection component has been performed using the spectra at rotational maximum and minimum, keeping constant the temperature, abundances, interstellar and partial covering hydrogen column densities at the values found from 

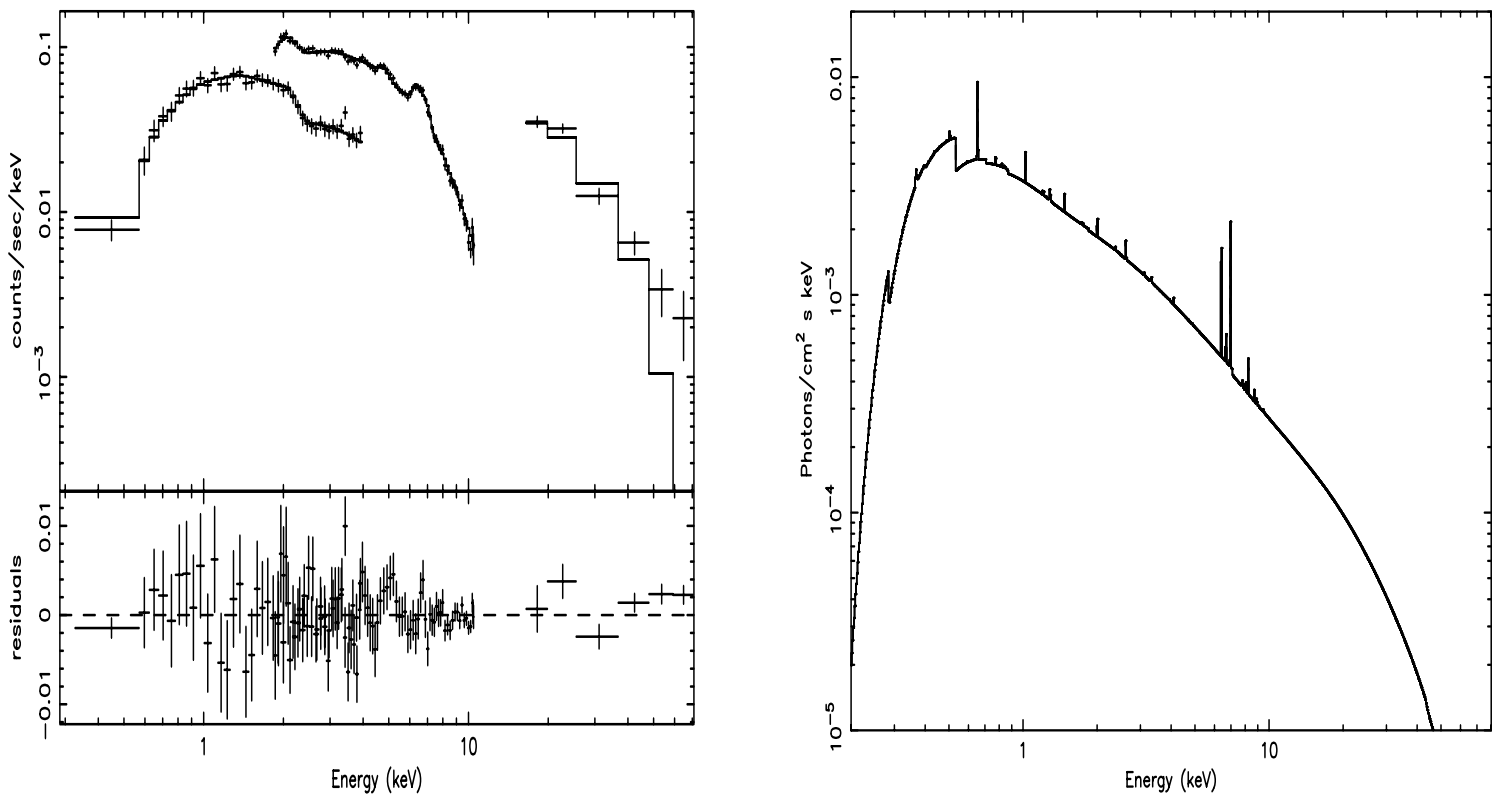

Fig. 6. Left panel: the grand average BeppoSAX LECS, MECS and PDS spectra fitted with an optically thin plasma at $k T=27 \mathrm{keV}$ together with an interstellar and partial covering absorbers and a Compton reflected continuum and Gaussian centred at $6.4 \mathrm{keV}$ (see text). Right panel: the X-ray spectrum corresponding to the composite model is displayed in physical units.

Table 2. Best fit parameters for the phase-averaged X-ray spectra of V 709 Cas.

\begin{tabular}{|c|c|c|c|c|c|c|c|c|c|c|c|}
\hline Data & $\#$ & $\begin{array}{c}N_{\mathrm{H}} \\
\left(10^{20} \mathrm{~cm}^{-2}\right)\end{array}$ & $\begin{array}{c}N_{\mathrm{H}}^{a} \\
\left(10^{22} \mathrm{~cm}^{-2}\right)\end{array}$ & $C_{\mathrm{F}}^{b}$ & $\begin{array}{c}k T^{c} \\
(\mathrm{keV})\end{array}$ & $A_{\mathrm{Z}}^{d}$ & $\begin{array}{l}E W^{e} \\
(\mathrm{eV})\end{array}$ & $R_{\mathrm{ref}}^{f}$ & $\tau$ & $\begin{array}{c}E_{\mathrm{o}} \\
(\mathrm{keV})\end{array}$ & $\chi^{2} /$ d.o.f. $\left(\chi_{\nu}^{2}\right)$ \\
\hline SAX & 1 & $8.7_{-2.9}^{+7.5}$ & $2.9_{-0.9}^{+1.6}$ & $0.29_{-0.05}^{+0.06}$ & $42_{-5}^{+6}$ & $1.29_{-0.29}^{+0.35}$ & $218_{-43}^{+22}$ & & & & $131 / 111(1.18)$ \\
\hline SAX & 2 & $8.0_{-2.8}^{+3.0}$ & $2.3_{-0.7}^{+0.9}$ & $0.30_{-0.03}^{+0.03}$ & $27_{-4}^{+6}$ & $0.73_{-0.20}^{+0.28}$ & $179_{-45}^{+25}$ & $0.9_{-0.5}^{+0.4}$ & & & $121 / 110(1.10)$ \\
\hline RXTE & 3 & & $1.6_{-0.1}^{+0.2}$ & $0.99_{-0.53}^{+0.01}$ & $36_{-2}^{+2}$ & $0.52_{-0.08}^{+0.09}$ & $216_{-16}^{+15}$ & & $0.13_{-0.02}^{+0.02}$ & $8.1_{-0.2}^{+0.1}$ & $45 / 37(1.23)$ \\
\hline RXTE & 4 & & $1.7_{-0.2}^{+0.1}$ & $0.99_{-0.55}^{+0.01}$ & $26_{-4}^{+6}$ & $0.40_{-0.17}^{+0.11}$ & $198_{-17}^{+10}$ & $0.3_{-0.2}^{+0.2}$ & $0.11_{-0.02}^{+0.02}$ & $8.0_{-0.2}^{+0.2}$ & $40 / 36(1.11)$ \\
\hline
\end{tabular}

${ }^{a}$ Column density of the partial absorber.

${ }^{b}$ Covering fraction of the partial absorber.

${ }^{c}$ Plasma temperature.

${ }^{d}$ Metal abundance in units of the cosmic value (Anders \& Grevesse 1989).

${ }^{e}$ Equivalent width of the $6.4 \mathrm{keV}$ fluorescent iron line.

${ }^{f}$ Relative normalization of the reflection component (see text).

fit with model 2 , in Table 2 . The variations of the relative normalization $R_{\text {refl }}$ in these fits are consistent with the iron line $E W$ behaviour shown in Fig. 7, being lower at rotational maximum $\left(R_{\text {refl }} \leq 0.2\right)$ and larger at minimum $\left(R_{\text {refl }}=0.7 \pm 0.4\right)$.

\subsection{The RXTE spectra}

The time-averaged RXTE spectrum was fitted first with a MEKAL model, together with a Gaussian line centred at $6.4 \mathrm{keV}$, and two absorbers to account for the interstellar and partial covering but the fit quality is bad $\left(\chi_{\nu}^{2}=5.97\right)$. The residuals show a strong excess of counts around $5 \mathrm{keV}$ and a depression around $8 \mathrm{keV}$. The presence of this feature has been checked against instrumental (background subtraction) effects, and in the individual spectra of all five PCU units. We interpret this feature as a signature of an absorption edge. Furthermore the interstellar column density results are negligible, as expected by the limited extension to low energies. The covering fraction of the partial absorber results instead close to unity (total absorber). Hence the fits have been performed again neglecting the interstellar absorption and introducing an additional component $\left(\mathrm{e}^{-\tau\left(E / E_{\mathrm{o}}\right)^{-3}}\right.$ at $\left.E>E_{\mathrm{o}}\right)$ in order to account for the absorption edge. As shown in Table 2 (model 3$)$, the fit quality substantially improves $\left(\chi_{\nu}^{2}=1.23\right)$. Except for the temperature $(k T=36 \mathrm{keV})$, some large differences are encountered in the spectral parameters with respect to the BeppoSAX results. First of all the presence of the edge with $E_{\mathrm{o}}=8.1 \mathrm{keV}$ and an optical depth $\tau=0.13$, which is not required, although not excluded by the BeppoSAX 


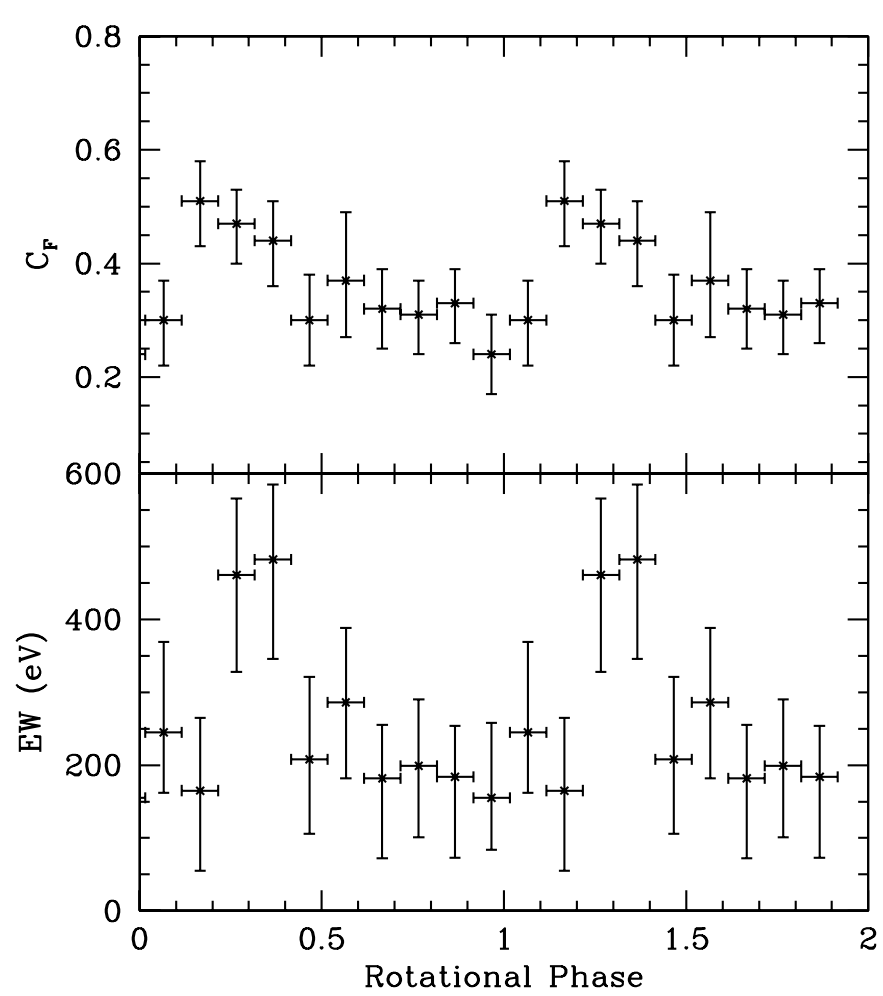

Fig. 7. Variations of the covering fraction of the partial absorber and $E W$ of fluorescence iron line along the spin pulse period obtained from the BeppoSAX observation. A composite model consisting of a MEKAL emission plus interstellar hydrogen column density, partial absorber and Gaussian line has been fitted to the combined LECS and MECS spectra extracted in 10 spin bins. The temperature, the interstellar and partial absorber column densities and abundances have been kept constant (see text).

data. Here we note that although BeppoSAX has a better energy resolution than RXTE, the latter has a much larger collecting area. The edge energy corresponds, within the error, to Fe XIX-XXII; the optical depth to an equivalent hydrogen column density of $\sim 1.1 \times 10^{23} A_{\mathrm{Fe}}^{-1} \mathrm{~cm}^{-2}$. The abundances results seem to be much lower than solar values. We note that fixing them to the solar value, the fit quality decreases noticeably $\left(\chi_{\nu}^{2}=2.13\right)$. The origin of such large difference is unclear, but it should be noted that at the low resolution of RXTE the various iron lines are heavily blended. Also, the absorber is total while column densities are within errors comparable to the BeppoSAX fits. The presence of such an absorber could be consistent with the presence of the absorption edge. The equivalent width of the $\mathrm{K}_{\alpha}$ iron line $(216 \mathrm{eV})$ is similar to that derived from the BeppoSAX data. With this model the observed flux in the $2-10 \mathrm{keV}$ range is $6.64 \times 10^{-11} \mathrm{erg} \mathrm{cm}^{-2} \mathrm{~s}^{-1}, \sim 1.4$ times larger than that observed in July 1998 by BeppoSAX. An attempt to fit the RXTE data with an additional reflection component has been performed, resulting in a modest improvement in the quality of the fit, significant at the $95.9 \%$ confidence level ( $F$-test; see Table 2 model 4 shown in Fig. 8). The temperature is lower $(k T=26 \mathrm{keV})$ than that derived in model 3 and similar to that derived from BeppoSAX data. The relative normalization of the reflection component is comparable, within errors, to the BeppoSAX fits. In summary the RXTE grand average spectrum provides the first evidence of absorption from ionized material.

We also performed a phase-resolved spectral analysis of the RXTE data, limiting ourselves to study the maximum and minimum phases. Within the errors, no parameter changes between the two phases apart from the column density of the cold absorber, which is $1.37_{-0.24}^{+0.23} \times 10^{22} \mathrm{~cm}^{-2}$ at the maximum and $2.33_{-0.34}^{+0.16} \times 10^{22} \mathrm{~cm}^{-2}$ at the minimum.

\section{Discussion}

The RXTE and BeppoSAX observations have allowed, for the first time, a simultaneous characterization of the X-ray temporal and spectral behaviour of V 709 Cas.

\subsection{Variability}

It has been possible to confirm the rotational period of the WD at $312.75 \mathrm{~s}$ and to rule out the presence of any orbital and sideband periodicities. This system is therefore a typical disc accretor. The rotational modulation is remarkably non-sinusoidal compared to most IPs. The spin pulses are clearly observed at all energies up to $25 \mathrm{keV}$, with decreasing amplitudes from soft to hard X-rays, a typical behaviour of IPs. Furthermore, we have found that the power at the spin frequency and first and second harmonics changes with time: the spin pulsed fraction observed by BeppoSAX in July 1998 was twice that observed in February of the same year by ROSAT (comparison being done in the $0.1-2.4 \mathrm{keV}$ range) and about 1.2 times larger than that observed by RXTE in March 1997 ( comparing the $2-5 \mathrm{keV}$ range). Not only the amplitude but also the morphology of the rotational modulation has changed. The one observed by BeppoSAX is highly asymmetrical with a maximum possessing two peaks with different intensities in the hard band, but it is single-peaked in the soft (ROSAT-like) band. The latter contrasts with the double-peaked maximum (with similar intensities) as observed by ROSAT. The BeppoSAX pulsation also differs from the very symmetrical RXTE spin pulse which presents a relatively flat-top maximum and more resembles the ROSAT light curve. It is worth noting that the pulse maximum extends over the same phase range in all observations. The presence of the first harmonic in the two observations in 1998, contrasts with the lack of detection in March 1997. A strong first harmonic, compared to the second, would produce a double peaked light curve with two maxima offsetted in phase by $180^{\circ}$. In V 709 Cas the second harmonic is the stronger and produces an asymmetrical profile when the first harmonic is present and strong, as observed by BeppoSAX. When the first harmonic is weak or even absent the curve possesses similar maxima as observed by ROSAT and RXTE. 

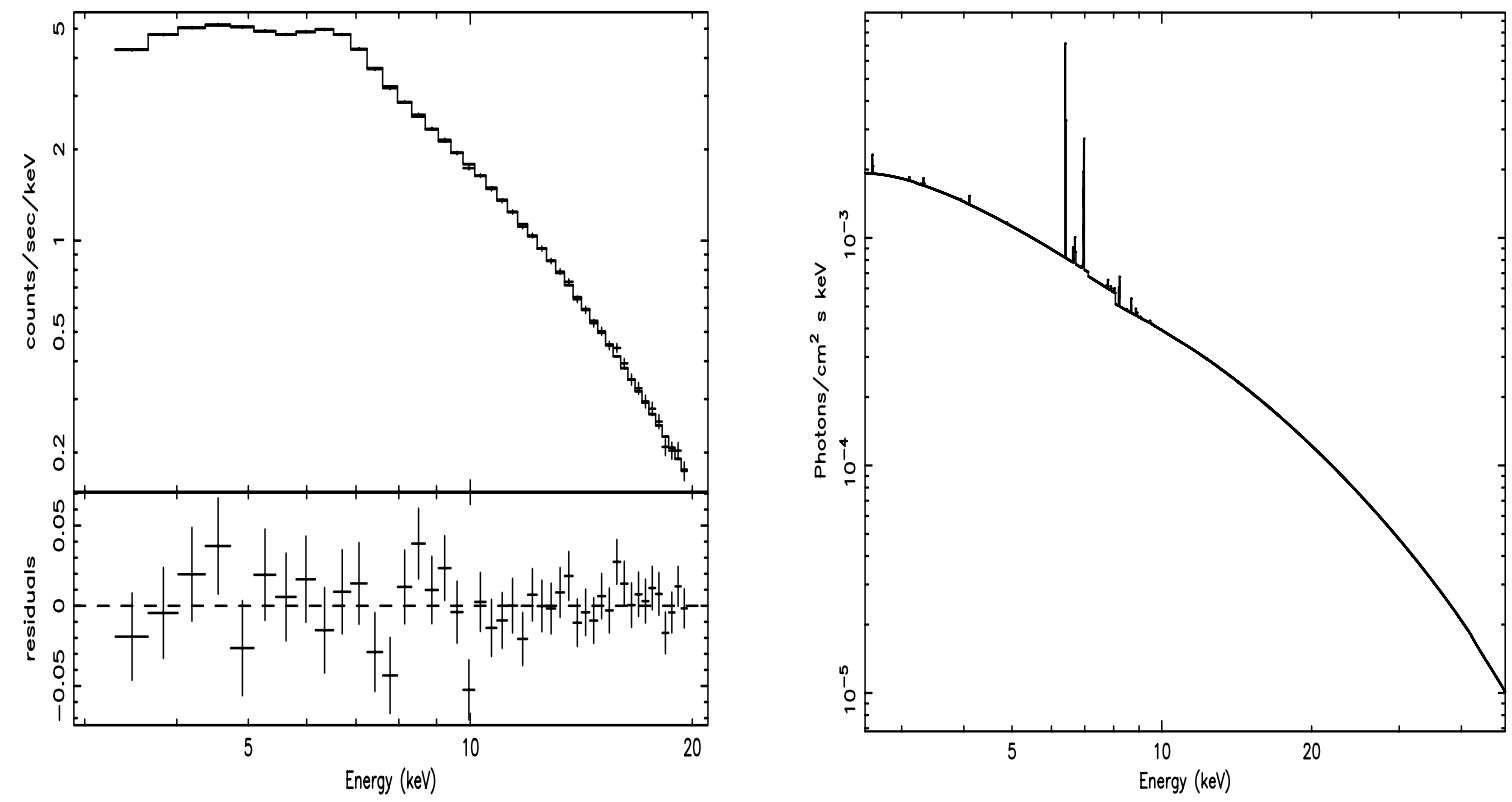

Fig. 8. Left panel: the grand average RXTE spectrum fitted with an optically thin plasma at $k T=26 \mathrm{keV}$ together with an absorber with $N_{\mathrm{H}}=1.7 \times 10^{22} \mathrm{~cm}^{-2}$, an absorption edge with $\tau=0.11$ and $E_{\mathrm{o}}=8.0 \mathrm{keV}$, and a Compton reflection continuum plus a Gaussian line centred at $6.4 \mathrm{keV}$ (model 4). Right panel: the X-ray spectrum corresponding to the composite model is displayed in physical units.

The changes in the shape of light curve are also accompained by variations in the X-ray flux. Comparing the ROSAT PSPC count rates observed in 1992 (Motch et al. 1996) with those converted from the ROSAT HRI pointing in February 1998 (Norton et al. 1999), we find that V 709 Cas was brighter by a factor of $\sim 1.2$ in the latter observation. Furthermore converting the BeppoSAX flux in the ROSAT energy pass-band, we find that the binary was brigher by a factor of $\sim 1.7$ in July of the same year. This suggests that V 709 Cas experiences changes in luminosity on time scales of less than months to years and supports the identification of a highly variable X-ray source made by Motch et al. (1996). Hence changes in the accretion rate are likely to occur in this system (see Sect. 5.2) which give rise to changes in the amplitude and shape of spin pulses. This phenomenon has also been observed in other IPs such as FO Aqr (Beardmore et al. 1998; de Martino et al. 1999), TX Col (Norton et al. 1997) and BGCMi (de Martino et al. 1995). As the accretion disc extends down to the magnetospheric radius, which scales as $\propto \dot{M}^{-2 / 7}$, an increase in the mass accretion rate can produce a shrinking of the magnetospheric radius and hence an accretion spread over larger areas of the WD surface. At higher accretion rates the effects of absorption are expected to be larger, thus influencing the shapes and amplitudes of the low energy rotational modulation. The increase in the spin amplitude between the February and July 1998 could be consistent with this interpretation. On the other hand, the March 1997 RXTE spin pulse has a lower amplitude with respect to that in 1998 . At highest accretion rates, some of the local absorbers become ionized, which can reduce the low energy modulation amplitudes.
The double-humped light curve observed by ROSAT was attributed to the contributions of both accreting poles by Norton et al. (1999), but with an offset of the dipole axis thus producing two maxima separated by less than half a spin period. In the two-pole accretion scenario there are two ways to produce a double-peaked spin pulsation. One invokes large areas, due to the low magnetic field of the accreting WD, with vertical optical depth (along field lines) lower than the horizontal optical depth. The other invokes both wide and tall shocks, but with the vertical optical depth larger than the horizontal, as in the classical accretion scenario. The RXTE and BeppoSAX observations have shown that the spin pulse is energy dependent and the pulses are in phase at all energies (i.e. above and below $10 \mathrm{keV}$ ). To account for a rotational modulation above $10 \mathrm{keV}$, reflection cannot be the main mechanism, since, as shown in Sect. 4, it would produce an anti-phased pulse with respect to the low energy pulsation. A self occultation of tall shocks was proposed as a solution for the high energy rotational modulation in IPs (Mukai 1999). This can account for the phasing of spin pulses at both high and low energies, for the lack of an energy dependence of the hard X-rays pulsations and for the sharpness of the rise and decay of the pulsation. Hence the possibility that in V 709 Cas the two accreting poles are wide and tall, with the optical depth along the magnetic field lines larger than across them (as in the classical accretion curtainmodel), seems to be the most viable. Since V 709 Cas is a classical disc-accretor, the accretion is expected to occur onto both poles. Also, in order to have the same phasing at both high and low energies, $i+\delta<90^{\circ}$, where $i$ is the binary inclination and $\delta$ is the magnetic 
colatitude (Mukai 1999), with an upper limit to the inclination of $i<68^{\circ}$ due to the lack of an X-ray eclipse in this system. This would imply that the upper pole dominates the hard X-rays at all phases and hence does not produce a strong modulation, but the lower pole produces a maximum when the upper pole points away from the observer. The lower pole appears into view for $\sim 40 \%$ of a cycle, thus producing the sharp rise to and sharp decay from the maximum. When the upper pole points towards the observer (spin minimum), the lower pole is out of view. A simplified sketch of the geometry is shown in Fig. 9 for an illustrative set of values, $i=45^{\circ}, \delta=30^{\circ}$ and shock height $h=0.2 R_{\mathrm{wd}}$ for two symmetrical poles. An asymmetry between the two poles was pointed out by Norton et al. (1999) which seems to be necessary to explain the details of the light curves. An offset value of $\sim 108^{\circ}$ could be derived from the separation of the two maxima in the ROSAT HRI light curve. However for a detailed modeling of the accretion geometry one should take into account the whole three-dimensional shape of the emission region which would imply too many degrees of freedom. For our purposes, we only note that the net effects of the absorption within the two intervening accretion curtains at their respective spin maximum will then harden the spectrum at these phases. This explains the lack of spectral changes during the maximum and dip phases. Furthermore, the BeppoSAX observation shows that this maximum is highly asymmetrical, which would suggest that the contribution from some parts of the accretion regions are not identical at some epochs.

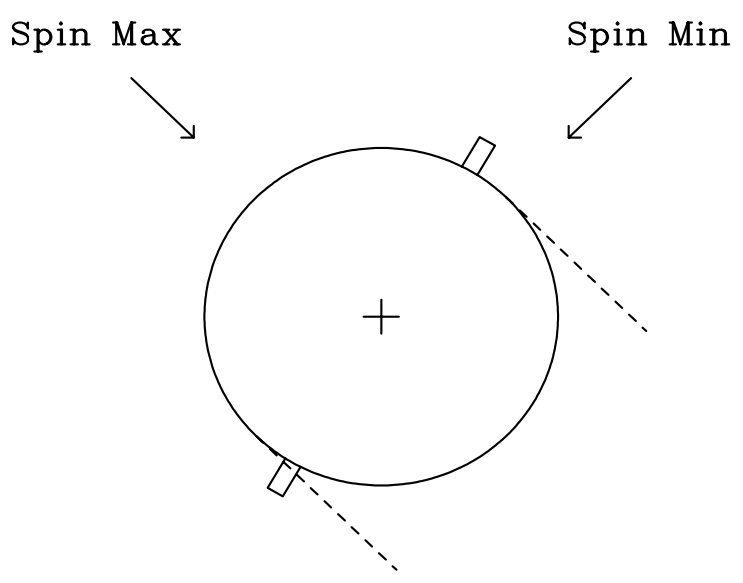

Fig. 9. A schematic sketch of the X-ray spin modulation in V 709 Cas for $i=45^{\circ}, \delta=30^{\circ}$ and a shock height of $0.2 R_{\mathrm{wd}}$ assuming for semplicity two symmetrical accreting poles. At spin minimum (the observer's line of sight is indicated by the arrow from the upper right), the lower pole is hidden, while the upper pole is seen along the field lines. At spin maximum (the line of sight is shown from the left), the entire upper pole and most of the lower pole are visible (the regions hidden by the WD are indicated by the dashed lines), and both poles are seen across the field lines. The lower pole is visible for $\sim 40 \%$ of a cycle, thus being responsible for the broad maximum. The upper pole does not produce a strong modulation, being visible at all spin phases.

\subsection{Spectral properties}

The spectral analysis carried out from 0.1 to $100 \mathrm{keV}$ has shown that V 709 Cas is a classical IP in its X-ray characteristics. A model consisting of a single temperature optically thin emitting gas, attenuated by both interstellar material and a partial absorber, and an emission feature corresponding to the fluorescent $6.4 \mathrm{keV}$ iron line, describes well the BeppoSAX X-ray spectrum. The post-shock temperature ranges between 42 and $27 \mathrm{keV}$, depending on whether reflection from the WD surface is included in the spectral fits. Within errors the difference between the two temperatures is not significant. We also note that a multi-temperature plasma is not required by either RXTE or BeppoSAX data. In this respect, we compare the derived temperature of the post-shock region with that expected from the simple shock model

$k T_{\text {shock }}=3 / 8 G \mu m_{\mathrm{H}} M_{\mathrm{wd}} R_{\mathrm{wd}}^{-1}$

with $\mu$ the mean molecular weight and $m_{\mathrm{H}}$ the mass of hydrogen. From optical spectroscopy, Bonnet-Bidaud et al. (2001) constrain the WD radius at a value $\sim 8.7 \times 10^{8} \mathrm{~cm}$ and a mass of $0.75 M_{\odot}$, which then implies a temperature of $30 \mathrm{keV}$, in close agreement with our BeppoSAX and RXTE results.

From the spectral fits we have found that the column density of the partial absorber cannot alone explain the large equivalent width $(\sim 200 \mathrm{eV})$ of the $6.4 \mathrm{keV}$ line. Hence the contribution from reflection by the cold material of the WD surface should dominate the fluorescent iron line. At a temperature of $\sim 30 \mathrm{keV}$, an $E W \geq 100 \mathrm{eV}$ is expected for $\theta<36^{\circ}$ (Matt 1999), where $\theta$ is the viewing angle between the line of sight and the direction orthogonal to the illuminated portion of the WD surface. From Fig. 9, the largest contribution to the iron line from the reflected region of the WD surface should then be produced by the upper pole at spin minimum $\left(\theta=15^{\circ}\right)$. However if some asymmetry exists, this enables to see the lower pole more effectively, thus giving rise to a large equivalent width also at other spin phases. We have indeed found spectral variations with the rotational period, which indicate a spin variability of the partial absorber and emission line parameters, the largest contribution of both being at rotational minimum. Again, the accretion curtain scenario matches well the spectral behaviour of spin pulses. When the curtain points towards the observer, the absorption effects are larger (the covering factor is larger) as well as the effects produced by reflection, since the accretion area projected on the WD is larger. This is also seen from fitting the spectra at spin maximum and minimum which show an increase in the Compton reflection component at spin minimum.

The spectral analysis of 1997 RXTE data has furthermore shown the presence of an absorption feature at $\sim 8.1 \mathrm{keV}$ which is interpreted as an absorption edge from Fe XIX-XXII with an optical depth $\tau \sim 0.11-0.13$. This would imply an ionization parameter of $\sim 100$ (Kallman \& Mc Cray 1982). This is the first detection of an 
ionized absorber in an IP. Such material could be located in the pre-shock regions of the accretion funnel. The lack of detection of a variability of this feature at the rotational period might suggest that this material is distributed over a large angle as seen by the observer. The results of the RXTE spectral fits also suggest the presence of a total absorber, which is consistent with a large covering area.

We attempt to obtain estimates of the accretion rate from the $\mathrm{X}$-ray luminosities of BeppoSAX and RXTE observations. Bonnet-Bidaud et al. (2001) constrain V709 Cas at a distance between 210-250 pc, which would imply a bolometric luminosity of $1.1-1.5 \times$ $10^{33} \mathrm{erg} \mathrm{s}^{-1}$ during the March 1997 RXTE observations and of $0.8-1.1 \times 10^{33} \mathrm{erg} \mathrm{s}^{-1}$ during the July 1998 BeppoSAX observations. This gives an accretion rate of $9.6-13.0 \times 10^{15} \mathrm{~g} \mathrm{~s}^{-1}$ in 1997 and of $6.9-9.6 \times 10^{15} \mathrm{~g} \mathrm{~s}^{-1}$ in 1998 . The accretion rate has then decreased by a factor of $\sim 1.4$ between 1997 and 1998. Hence the detection of an ionized material in the RXTE data could be consistent with a higher accretion rate experienced in 1997.

We also derive an average emission measure $E M=$ $2.6-3.8 \times 10^{55} \mathrm{~cm}^{-3}$ and $E M=1.7-2.4 \times 10^{55} \mathrm{~cm}^{-3}$ for the March 1997 and July 1998 observations, assuming the above range of distances. As $E M \sim n_{\mathrm{H}}^{2} \times l^{3}$, we give an upper limit to the number density of hydrogen and an estimate to the linear dimension of the post-shock region. We use the RXTE results since from the iron absorption edge, we have derived the equivalent hydrogen column density $N_{\mathrm{H}} \sim 1.1 \times 10^{23} A_{\mathrm{Fe}}^{-1} \mathrm{~cm}^{-2}$. Assuming that this material is in the pre-shock region, and for simplicity in the immediate vicinity of the shock, the density in the region just below the shock is a factor of four higher. Then, from the range of emission measure, we obtain $l \sim 3.4-4.9 \times 10^{7} \mathrm{~cm}$ and $n_{\mathrm{H}} \sim 1.8-2.6 \times 10^{16} \mathrm{~cm}^{-3}$. The former gives a nonnegligible shock height consistently with a tall shock as suggested from the spin light curve.

\section{Conclusion}

The BeppoSAX and RXTE observations have allowed for the first time the study of the broad band X-ray emission spectrum and variability of V 709 Cas which can be characterized as follows:

a) V 709 Cas is dominated by the rotational pulsation of the accreting WD at a 312.75 s period, with no sign of orbital or sideband periodicities. This confirms that it is a disc accretor.

b) Its spectrum is hard and well described by an isothermal optically thin plasma at $27 \mathrm{keV}$ with complex absorption and an iron $\mathrm{K}_{\alpha}$ fluorescent line, due to reflection from the WD surface. Evidence of cool and ionized absorbing material in the pre-shock region is found in this system, e.g. from a partial absorber and an iron absorption edge.

c) The rotational pulsation is compatible with complex absorption dominating the low energy range, while at higher energies pulsation is likely due to the presence of a non-zero shock height above the accreting poles.

d) Variations along the rotational pulse in the partial covering absorber and reflection are compatible with the classical curtain scenario, where accretion material flows from the disc towards the polar regions of the WD via arc-shaped curtains.

e) The shape and amplitude of the spin light curve as well as the X-ray flux change with time indicating that V709 Cas experiences variations in the mass accretion rate on timescales of less than months to years.

Acknowledgements. We gratefully acknowledge Prof. J. Patterson for the usage of the RXTE data. We also wish to thank Dr. C. Done for kindly providing the reflection fitting code. The BeppoSAX team and Science Data Center staff are warmly thanked for their help and advice in the data handling and reduction. We also acknowledge useful discussion with the RXTE team, Dr. J. Swank and Dr. K. Jahoda, on PCA instrument calibration and data analysis. D. dM. and G. M. acknowledge financial support from ASI.

\section{References}

Anders, E., \& Grevesse, N. 1989, Geochimica et Cosmochimica Acta 53, 197

Beardmore, A. P., Done, C., Osborne, J. P., et al. 1995, MNRAS, 272, 749

Beardmore, A. P., Mukai, K., Norton, A. J., et al. 1998, MNRAS, 297, 337

Boella, G., Butler, R. C., Perola, G. C., et al. 1997, A\&AS, 122, 299

Bonnet-Bidaud, J.-M., Mouchet, M., de Martino, D., et al. 2001, A\&A, 374, 1003

Bradt, H. V., Rothschild, R. E., \& Swank, J. H. 1993, A\&AS, 97, 355

Buckley, D. A. H. 1996, in Proc. Cataclysmic Variables and Related Objects, ed. A. Evans, \& J. H. Wood, IAU Coll. 158, Space Sci. Lib. 208, 185

Buckley, D. A. H., Haberl, F., Motch, C., et al. 1997, MNRAS, 287,117

Deeming, T. J. 1975, Ap\&SS, 36, 137

Done, C., Mulchaey, J. S., Mushotzky, R. F., et al. 1992, ApJ, 395,275

Done, C., Osborne, J. P., \& Beardmore, A. P. 1995, MNRAS, 276,483

de Martino, D. 1993, in Proc. 2nd Haifa Conference on Cataclysmic Variables and related physics, ed. O. Regev, \& G. Shaviv, 201

de Martino, D., Mouchet, M., Bonnet-Bidaud, J. M., et al. 1995, A\&A, 298, 849

de Martino, D., Silvotti, R., Buckley, D. A. H., et al. 1999, A\&A, 350, 517

Haberl, F., \& Motch, C. 1995, A\&A, 297, L37

Hellier, C. 1995, in ASP Conf. Ser. 85, Cape Workshop on Magnetic Cataclysmic Variables, ed. D. A. H. Buckley, \& B. Warner, 185

Hellier, C. 1999, in ASP Conf. Ser. 157, Annapolis Workshop on Magnetic Cataclysmic Variables, ed. C. Hellier, \& K. Mukai, 1

Inoue, H. 1985, SSRv, 40, 317

Ishida, M., Mukai, K., \& Osborne, J. P. 1994, PASJ, 46, L81 
Kallman, T., \& Mc Cray, R. 1982, ApJSS, 50, 263

Kozhevnikov, V. P. 2001, A\&A, 366, 891

Matt, G. 1999, in ASP Conf. Ser. 157, Annapolis Workshop on Magnetic Cataclysmic Variables, ed. C. Hellier, \& K. Mukai, 299

Matt, G., Perola, G. C., \& Piro, L. 1991, A\&A, 247, 25

Mewe, R., Kaastra, J. S., \& Liedahl, D. A. 1995, Legacy (Journal of HEASARC), 6, 16

Motch, C., Haberl, F., Guillout, P., et al. 1996, A\&A, 307, 459

Mukai, K., in ASP Conf. Ser. 157, Annapolis Workshop on Magnetic Cataclysmic Variables, ed. C. Hellier, \& K. Mukai, 33

Norton, A. J., Beardmore, A. P., \& Taylor, P. 1996, MNRAS, 280937
Norton, A. J., Hellier, C., Beardmore, A. P., et al. 1997, MNRAS, 289, 362

Norton, A. J., Beardmore, A. P., Allan, A., et al. 1999, A\&A, 347,203

Patterson, J. 1994, PASP, 106, 209

Roberts, D. H., Lehár, J., \& Dreher, J. W. 1987, AJ, 93, 968

Rosen, S. R., Mason, K. O., \& Cordova, F. A. 1988, MNRAS, 231, 549

Singh, K. P., White, N. E., \& Drake, S. A. 1996, ApJ, 456, 766

Wynn, G., \& King, A. 1992, MNRAS, 255, 83

Warner, B. 1986, MNRAS, 219, 347

Warner, B. 1995, Cataclysmic Variable Stars (Cambridge Univ. Press, Cambridge) 